\title{
PARÁSITOS EN PLAYAS TURÍSTICAS: PROPUESTA DE INCLUSIÓN COMO INDICADORES DE CALIDAD SANITARIA. REVISIÓN PARA AMERICA LATINA
}

\section{PARASITES IN TOURIST BEACHES: PROPOSAL FOR ITS INCLUSION AS HEALTH QUALITY INDICATORS. REVIEW FOR LATIN AMERICA}

\author{
Ganiveth Manjarrez ${ }^{1}$, Jorge Blanco², Betsy González ${ }^{3}$, Camilo M. Botero ${ }^{4}$ y Claudia Díaz-Mendoza ${ }^{5}$
}

\section{Resumen}

Las playas, además de ser centro de recreación turística, contienen gran cantidad de bioma microscópica con diferentes especies. La mayoría de agentes patógenos están presentes en la arena, ocasionando alta probabilidad de infección humano - parásito, especialmente por contacto directo y hábitos higiénicos deficientes. Así mismo, el estrecho vínculo que se da entre animales y humanos crea el ambiente propicio para el surgimiento de zoonosis de importancia en salud pública, especialmente las relacionadas con animales de compañía como perros y gatos. Los monitoreos que generalmente se realizan en playas turísticas incluyen como indicadores de calidad sanitaria a Coliformes totales, Coliformes fecales y en algunos casos a enterococos, excluyendo la búsqueda de parásitos como parte de los parámetros que definen el estado sanitario de las playas. En consecuencia, los resultados no reflejan integralmente las condiciones ambientales de las playas y el riesgo de afectación a la salud humana. En esta revisión se analizaron cuarenta y dos artículos (42) de América Latina, así como literatura especializada de Europa y Estados Unidos, en los cuales se presentan argumentos que respaldan la inclusión permanente de parásitos como indicadores de calidad sanitaria tales como Ancylostoma sp., Strongyloides sp. y Taxocara sp., demostrando la importancia de su determinación, principales especies indicadoras, técnicas de laboratorio para su aislamiento y estudios recientes relacionados con el tema.

Palabras clave: arena, calidad sanitaria, parásitos, playas turísticas.

\begin{abstract}
The beaches, besides being a tourist recreation center, contain a large amount of microscopic biome with different species. Most pathogens are present in the sand, causing a high probability of human-parasite infection, especially by direct contact and poor hygienic habits. Likewise, the close bond that exists between animals and humans creates an environment conducive to the emergence of zoonoses of importance in public health, especially those related to companion animals such as dogs and cats. The monitoring usually carried out on tourist beaches includes as indicators of sanitary quality, total coliforms, fecal coliforms and in some cases enterococci, excluding the search for parasites as part of the parameters that define the sanitary status of the beaches. Consequently, the results do not fully reflect the environmental conditions of the beaches and the risk of affecting human health. In this review, forty-two articles (42) from Latin America were analyzed, as well as specialized literature from Europe and the United States, in which arguments are presented that support the permanent inclusion of parasites as indicators of health quality such as Ancylostoma sp. Strongyloides sp. and Taxocara sp., demonstrating the importance of their determination, main indicator species, laboratory techniques for their isolation and recent studies related to the topic. Key words: sand, sanitary quality, parasites, tourist beaches.
\end{abstract}

\section{Introducción}

El concepto calidad ambiental en playas turísticas está relacionado con la seguridad física que estos ambientes costeros representan para los usuarios, asociado a los riesgos para la salud humana (Benedict \& Neumann, 2004; Herrera \& Suarez, 2005; Elmir et al., 2007; Oigman-Pszczol \& Creed, 2007; Costa et al., 2009; Delgado et al., 2009; Mansilha et al., 2009). Esto se debe principalmente a que múltiples actividades recreativas en las playas involucran el contacto directo con el agua y la arena (Elmanama et al., 2005; Ariza et al., 2008; Phillips et al., 2011).

Una de las vías más comunes para medir la calidad ambiental en playas es a través de indicadores que representan los componentes naturales y de bienestar humano (Morgan, 1999; Cagilaba \& Rennie, 2005; Espejel et al., 2007; Botero et al., 2015a). Estos indicadores están compuestos por parámetros 
fisicoquímicos y biológicos (Vandermeulen \& Cobb, 2004; Tudor \& Williams, 2008; Hurtado et al., 2009), cuyos resultados de medición actúan como criterios para evaluar la calidad de las playas (Herrera \& Suárez, 2005; Vogel et al., 2007; Gavio et al., 2010; Zhang et al., 2013). En ese contexto, se considera como referente el enfoque propuesto por Botero et al. (2015b), quienes definen la calidad sanitaria como el riesgo de afectación a la salud humana por las condiciones ambientales de la playa.

Múltiples investigaciones han sido realizadas sobre microorganismos en agua de mar. Las bacterias coliformes totales y fecales son consideradas las principales indicadoras de contaminación fecal. Estos microorganismos pertenecen a la familia Enterobacteriaceae (Delgado et al., 2008; SantiagoRodriguez et al., 2012); los más respresentantivos son: Escherichia coli sp., Citrobacter sp., Enterobacter sp. y Klebsiella sp. (Salcedo, 2013). Otro indicador de materia fecal son los Enterococcus (Díaz et al., 2010; Vergaray et al., 2007). Según Díaz et al. (2010), la presencia de Enterococcus faecalis es considerada como indicador de contaminación fecal de fuentes humanas, mientras que Enterococcus faecium indica contaminación fecal de origen animal.

Los bañistas, en sus actividades de recreación, pasan la mayor parte del tiempo en la arena de la playa y los microorganismos son un componente significativo en este medio, actuando como reservorio de vectores y fuente de infecciones a usuarios (Madrid et al., 2005). Sin embargo, en países latinoamericanos como Argentina, Venezuela, Perú, Colombia, México, y Chile (Orellana, 2005; DIGESA, 2011), contemplan solo la determinación de coliformes totales, coliformes fecales y enterococos en aguas marinas de uso recreativo; mientras que la búsqueda de parásitos no está reglamentada. La presencia de parásitos (tanto protozoos como helmintos) en arena de playas puede ofrecer información importante acerca de su calidad, la cual está relacionada con la presencia de animales, heces fecales y residuos orgánicos (González y Cáceres et al., 2005). Por lo tanto, la identificación de parásitos de importancia sanitaria en las playas permitirá proporcionar recomendaciones que contribuyan a mejorar el uso recreativo de estas.

Como aporte a esta discusión y como elemento para investigaciones más profundas, este artículo presenta las principales especies parasitarias indicadoras de calidad sanitaria de arena de playas, compara técnicas para su aislamiento e identificación y presenta los avances al respecto en América Latina.

Indicadores tradicionales de calidad ambiental de playas

El "Programa de calidad ambiental en playas turísticas del Caribe, Norte colombiano" (Botero et al., 2015a) toma como referencia aspectos de salud, ecosistema y recreación. El estudio en mención seleccionó 22 parámetros entre fisicoquímicos, microbiológicos y de observación directa, de acuerdo a normas nacionales colombianas, junto aspectos medibles de calidad ambiental (Barbosa \& Ferreira, 2008; Gavio et al., 2010; Herrera \& Suárez, 2005; Vogel et al., 2007) y documentos internacionales oficiales de calidad de playas. Este programa agrupa los parámetros seleccionados en indicadores de calidad ecosistémica, recreativa y sanitaria.

El indicador de calidad ambiental sanitaria mide la adecuación de las condiciones de la playa para la salud humana. Los parámetros incluidos para medir este indicador en arena de playas incluyen vectores, residuos sólidos, coliformes totales, coliformes fecales y enterococos. Esta revisión propone la inclusión de parásitos presentes en arena de playas como indicadores de calidad ambiental sanitaria, dado que se comportan como bioindicadores, entendidos como las especies que responden a condiciones ambientales específicas y permiten detectar modificaciones tempranas y/o de origen difuso en los ecosistemas, asociándose a su condición de calidad (Roldán, 2003; Vandermeulen \& Cobb, 2004; Cagilaba \& Rennie, 2005; Rocca et al., 2008; Tudor \& Williams, 2008).

Entre los principales indicadores de calidad ambiental en playas turísticas están los coliformes totales y coliformes fecales, los cuales forman parte de la microbiota normal del intestino del ser humano y de los animales homeotermos. Los coliformes son un grupo heterogéneo de bacterias representado por los géneros Escherichia spp., Citrobacter spp., Enterobacter spp.y Klebsiella spp., cuya procedencia puede ser fecal (se encuentran en el intestino del hombre y de los animales), pero también pueden aislarse del suelo, polvo y agua. Por ello, cuando se requiere saber si la contaminación es de origen fecal, se recurre a los coliformes fecales, y más comúnmente, a E. coli (Espindula, 2004; Larrea et al., 2009).

El uso de estos microorganismos como indicadores de contaminación fecal se favorece debido al amplio rango de temperatura óptima de crecimiento (hasta $45^{\circ} \mathrm{C}$ ). Entre las limitaciones de estas bacterias, para indicar contaminación fecal, está su limitada capacidad para sobrevivir en cuerpos de agua, su limitada habilidad para multiplicarse después de su liberación en una columna de agua y la debilidad de su pared celular frente a los procesos de desinfección (Marchand, 2002; González et al., 2010; Méndez et al., 2010).

Los enterococos también son considerados indicadores de contaminación fecal, son capaces de resistir a condiciones ambientales adversas como congelación, desecación, altas concentraciones de sal, poca concentración de oxígeno y a temperaturas que oscilan entre $42.7^{\circ} \mathrm{C}$ y $47.8^{\circ} \mathrm{C}$; además, pueden existir en lugares ácidos y alcalinos, de manera que demuestran ser bacterias extremófilas (Van den Berghe et al., 2006; Yamahara et al., 2009; Díaz et al., 2010). Su uso como indicador de contaminación fecal de aguas recreativas fue recomendado por la Agencia de 
Protección Ambiental de los Estados Unidos (USEPA, de las siglas en inglés) en 1986 (EPA, 1986), por su relación con gastroenteritis, enfermedades respiratorias, conjuntivitis y dermatitis (Vergaray et al., 2007; Díaz et al., 2008; Efstratiou et al., 2009).

Los vectores también se constituyen en indicadores de calidad sanitaria en playas, debido a que su presencia en mascotas o animales callejeros favorece la transmisión de enfermedades infecciosas entre personas, o de animales a personas. (Botero et al., 2015b). Muchos de los vectores son insectos hematófagos que ingieren microorganismos patógenos junto con la sangre de un portador infectado (persona o animal) y posteriormente, los inoculan a un nuevo portador al ingerir su sangre. Los mosquitos son los vectores de enfermedades mejor conocidos, pero también se conocen como vectores las garrapatas, moscas, flebótomos, pulgas y triatominos. En todo el mundo se registran defunciones como consecuencia de enfermedades transmitidas por vectores, como el paludismo, dengue, esquistosomiasis, tripanosomiasis africana humana, leishmaniasis, enfermedad de Chagas, fiebre amarilla, encefalitis japonesa y oncocercosis. Las enfermedades transmitidas por vectores representan más del $17 \%$ de todas las enfermedades infecciosas alrededor del mundo, su distribución está determinada por factores medioambientales y sociales como desplazamientos, urbanización no planificada y problemas medioambientales, entre ellos el cambio climático (WHO, 2014).

Los residuos sólidos en arenas y agua de mar se constituyen en un parámetro significativo para la determinación de calidad ambiental sanitaria de playas turísticas. Según el Reglamento Técnico del Sector Agua potable y Saneamiento básico colombiano, también llamado RAS 2000 en Colombia (Ministerio de Vivienda, Ciudad y Territorio, 2012), un residuo sólido es cualquier objeto, material, sustancia o elemento que se abandona, descarta o rechaza después de haber sido consumido o usado en actividades domésticas, industriales, comerciales, institucionales, de servicios entre otras. Para el caso de residuos sólidos en playas, estos por sus características o disposición final, pueden llegar al agua litoral cuando son arrojados al agua de mar desde embarcaciones, transportándose con ayuda de las olas o de corrientes marinas hasta llegar a las costas, o por acción de las olas que rompen en la playa y arrastran los sólidos livianos. Los residuos más comunes son empaques plásticos, papel y restos vegetales. En la actualidad existe una metodología establecida para cualificar residuos sólidos en arenas, mediante unidades, cantidad y tipo de residuos. Esta metodología genera el porcentaje de distribución de residuos sobre la arena y permite establecer la probabilidad del riesgo que representa el residuo al usuario (Barbosa, 2006; Silva, 2008; Díaz \& Prada, 2015).
Parásitos presentes en arena de playas y su inclusión como indicadores de calidad ambiental sanitaria

Los parásitos son microorganismos que representan patogenicidad para el ser humano. Desde 1981, los protozoos entéricos como Giardia lamblia, Entamoeba histolytica, Balantidium coli y Cryptosporidium parvum son reconocidos como causantes de brotes infecciosos transmitidos por el agua. Este último es de importancia mundial entre todos los enteropatógenos de transmisión hídrica; así, se constituye en indicador fundamental de calidad de agua o playas (WHO \& UNICEF, 2005; Cermeño et al., 2008; Enríquez et al, 2010; Pullés, 2014; Fredes, 2015).

Los helmintos se caracterizan por su alta persistencia en el ambiente, mínima dosis infecciosa, baja respuesta inmune y la capacidad de permanecer en el suelo por largos periodos de tiempo (Steiner et al., 1997; Nelson, 2003), por lo que se constituyen en indicadores adecuados de calidad sanitaria (Pulido et al., 2005).

Por otra parte, la arena es el reservorio ideal para parásitos y permite alta probabilidad de infección al humano, favorecida por el contacto directo, los hábitos higiénicos deficientes y la presencia de animales (Benedict \& Neumann, 2004; Herrera \& Suárez, 2005; Madrid et al., 2005; Elmir et al., 2007; Oigman-Pszczol \& Creed, 2007; Costa et al., 2009; Delgado et al., 2009; Mansilha et al, 2009). Además, la arena crea el ambiente propicio para el surgimiento de las zoonosis de interés en salud pública, especialmente aquellas relacionadas con los animales de compañía perros y gatos (Passucci \&West, 1996; Schapiro et al., 2001; Madrid et al., 2005).

Los suelos arenosos representan una fuente importante de infección humana por parásitos, debido a sus características geológicas, sedimentos no consolidados susceptibles a transporte y variaciones de humedad. La Organización Mundial de la Salud sostiene que los turistas, en particular aquellos que visitan países tropicales y subtropicales, están expuestos a infecciones por helmintos intestinales, asociados con la falta de higiene que permiten la contaminación del suelo con heces humanas o caninas (WHO, 2012). Además, aquellos nematodos que penetran la piel, representan un riesgo para los turistas en países donde las playas están contaminadas con heces humanas y/o caninas (Madrid et al., 2005).

Las geohelmintiasis constituyen un grupo de infecciones parasitarias causadas por helmintos que requieren de la arena para satisfacer etapas de su ciclo de vida ya que los estados inmaduros de algunos parásitos presentes en perros son eliminados en las heces, contaminando el suelo circundante, por consiguiente, los huevos deben ser ingeridos y las larvas penetrar a través de la piel. El hombre se comporta como hospedador accidental, y puede desarrollar distintas patologías, dependiendo del agente etiológico (Cobas, 2005). 
Otra razón por las cual los parásitos se constituyen en indicadores adecuados de calidad sanitaria es su fácil identificación. Expertos sugieren que la supervisión de arena debe realizarse junto a los monitoreos regulares de agua, con protocolos estandarizados que permitan comparaciones apropiadas entre lugares de playa (Sabino et al., 2013).

Análisis de parásitos en arena de playas turísticas

Los monitoreos para la identificación de parásitos en playas constan de análisis in situ y análisis de laboratorio. Los procedimientos de toma de muestras de arena de playas con la finalidad de aislar microorganismos han sido descritos previamente por Morales \& Esquivia (2014). Estos mismos procedimientos pueden adaptarse para parásitos.

Las técnicas para la identificación de parásitos en laboratorio, a partir de muestras de arena, son diversas. Beltrán et al. (2003) y Girard de Kaminskyrina (2003) han reportado la utilidad de la técnica de sedimentación rápida o concentración por sedimentación sin centrifugación, la cual ha sido útil para la búsqueda de nematodos como Ascaris lumbricoides. La concentración por migración o método de Baermann también se recomienda para la identificación de trofozoítos y larvas en movimiento como Balantidium coli y larvas de Strongyloides stercoralis (Castillo \& Bermúdez, 2006; Coyne et al, 2007; Ortigoza \& Cruz, 2012).

Las técnicas de Faust, Parodi Alcaraz y Sloss Sheather, basadas en la capacidad de los quistes y/o huevos de parásitos para flotar en sulfato de zinc y azúcar respectivamente, son recomendadas para buscar quistes y ooquistes de protozoos y/o huevos de helmintos (Castillo \& Bermúdez, 2006; Mejía, 2010; Ortigoza \& Cruz, 2012; Mariano et al., 2012).

Parásitos frecuentemente encontrados en playas turísticas

Entre los parásitos más frecuentemente encontrados en arena de playas turísticas se encuentra Ancylostoma sp. Estos son parásitos cosmopolitas de regiones tropicales y subtropicales. Los parásitos adultos viven en el intestino delgado del huésped. La fuente de infección para el hombre son las arenas contaminadas con heces de gatos o perros infectados. Los suelos que retienen la humedad son los más favorables para su desarrollo (Cordero del Campiño \& Rojo, 1999; Rodríguez \& Cob, 2005; Polo, 2006).

Strongyloides sp. es un helminto antropopatógeno que puede replicarse dentro del huésped humano. Es un nematodo endémico de regiones geográficas tropicales, subtropicales y hasta templadas, donde se dan las condiciones adecuadas para su desarrollo (temperatura, humedad, materia orgánica y condiciones sanitarias deficientes). La infección comienza cuando las larvas presentes en la tierra penetran la piel de quienes caminan descalzos; atraviesan luego los capilares y viajan a los alvéolos pulmonares, ascienden por los bronquios, la tráquea y son deglutidas, llegan finalmente al duodeno-yeyuno, asentándose dentro de las criptas de Lieberkühnen la mucosa entérica (Carrada, 2008). La infección puede ser asintomática, pero existe una gran morbi-mortalidad en personas inmunocomprometidas, en sujetos desnutridos $\mathrm{y}$ pacientes con otras enfermedades que pueden desarrollar hiperinfecciones; el parásito tiene el potencial de producir autoinfección interna y multiplicarse en los seres humanos (Pichard et al., 2014).

Taxocara sp. es uno de los parásitos de mayor prevalencia en caninos y felinos a nivel mundial (Glickman, 1993; Magnaval et al., 2001). La presencia de felinos infectados con T. cati en espacios públicos, sus hábitos de defecación y el contacto directo de esta especie con el hombre, podrían suponer, al igual que con T. canis, un riesgo zoonótico (Dubinsky et al., 1995; Fisher, 2003). Estos parásitos tienen un ciclo con estadios de vida libre, en los que las condiciones ambientales y la presencia de hospedadores paraténicos desempeñan un papel fundamental en su dispersión (Dubinsky et al., 1995). Los huevos de Toxocaras sp. son altamente resistentes y su viabilidad y evolución en el ambiente dependen del tipo de suelo, la temperatura y la humedad $\left(25-35^{\circ} \mathrm{C}\right.$ y $85 \%$ de humedad) (Ludlam \& Platt, 1989; Shimizu, 1993; Overgaauw, 1997; Lescano et al., 1998; Alonso et al., 2001; Daprato et al., 2011).

\section{Materiales y métodos}

Para esta revisión, se consideraron las pautas propuestas por Kitchenham (2004) con algunas modificaciones. La metodología incluyó selección de revistas, definición de criterios de inclusión y exclusión y categorías de análisis.

Selección de revistas

Para la selección de cinco (5) revistas más relevantes, se utilizó la base de datos Scopus, usando la categoría parasites in beach como punto de partida.

En Scopus la opción compare Journal permite comparar las revistas a través del indicador de impacto SJR, el impacto por publicación IPP, el Impacto normalizado por artículo SNIP, las citaciones, los documentos fuentes por año, el porcentaje de documentos publicados no citados por año y el porcentaje de artículos de revisión publicados por año.

La Tabla 1 muestra las cinco revistas que publican estudios sobre parásitos en playas, con su factor de impacto.

Criterios de inclusión y exclusión

$\mathrm{Se}$ consideraron como criterios generales los estudios publicados entre 1984 y 2016 que describieran estudios de parásitos en playas. Como criterios específicos se definió seleccionar estudios que reportan la identificación de parásitos en playas y sus aplicaciones como indicadores de calidad.

Se excluyeron estudios no identificados como artículos en las revistas seleccionadas, por ejemplo: reseñas de 
Tabla 1. Factor de Impacto de revistas que publican estudios sobre parásitos en playas.

\begin{tabular}{|c|c|}
\hline Revista & $\begin{array}{l}\text { Factor de Impacto } \\
\text { (SJR) }\end{array}$ \\
\hline Journal of Parasitology & 0.695 \\
\hline Diseases of Aquatic Organisms & 0.964 \\
\hline Veterinary Parasitology & 1.213 \\
\hline $\begin{array}{l}\text { Chinese Journal } \\
\text { Schistosomiasis Control }\end{array}$ & 0.134 \\
\hline Marine Pollution Bulletin & 1.264 \\
\hline
\end{tabular}

libros, reseñas de capítulos de libros, editoriales o cartas al editor.

El análisis de contenido de los artículos permitió codificarlos y clasificarlos de acuerdo a los criterios previamente definidos, para encontrar las tendencias de investigación en parásitos en playas y sus aplicaciones como indicadores de calidad.

\section{Resultados y discusión}

Tomando como criterios los países con investigaciones en el tema, el número de artículos científicos publicados y el desarrollo de innovaciones relacionadas con el tema, se identifica en la base de datos Scopus el creciente aumento en publicaciones indexadas relacionadas con la temática de estudio, registrándose un máximo de cinco (5) publicaciones en el año 2014, tres (3) en los años 2006, 2009, 2015 y 2017, y dos (2) en los años 2005, 2007 y 2008. La Figura 1 presenta la tendencia en publicaciones relacionadas con calidad ambiental sanitaria y parásitos en arena de playas en un lapso de 18 años, desde el 2000 hasta el 2018.

Las subáreas en las que se desarrollan con mayor frecuencia investigaciones relacionadas con calidad sanitaria ambiental en playas son: agricultura y ciencias biológicas, ingeniería ambiental, medicina e inmunología, enmarcadas principalmente en áreas del

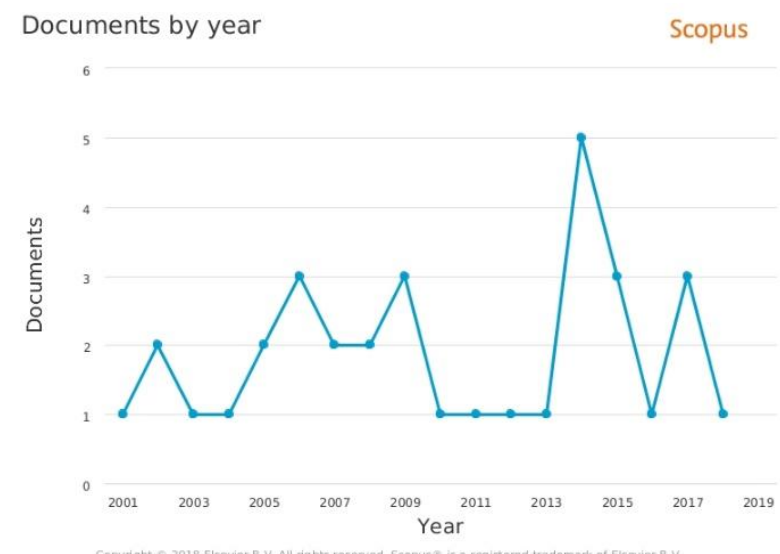

Figura 1. Tendencia en publicaciones relacionadas con indicadores sanitarios de calidad ambiental en playas entre los años 2000 a 2018 Fuente: Los autores a partir de www.elsevier.com.
Documents by subject area Scopus

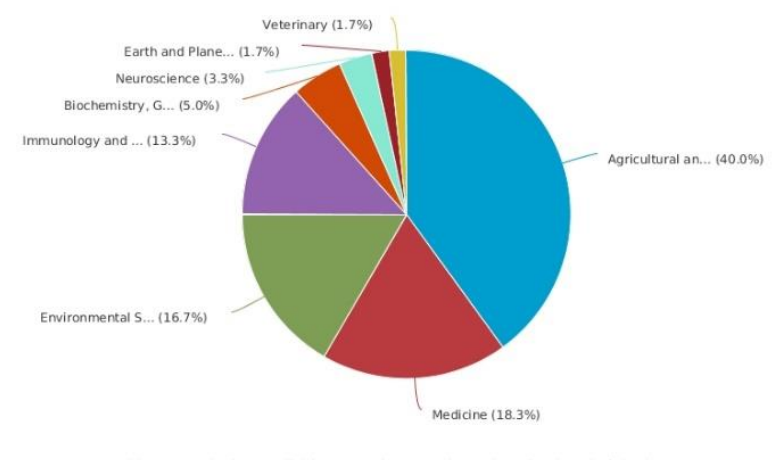

Figura 2. Subáreas en las que se desarrollan con mayor frecuencia investigaciones relacionadas con indicadores sanitarios (parásitos en arena de playa) de calidad ambiental en playas.

conocimiento de agricultura y ciencias biológicas e Ingeniería. La Figura 2 presenta la sub áreas en las que se desarrollan con mayor frecuencia este tipo de investigaciones. La sub área de agricultura y biología incluyen interacciones de parásitos con otros organismos en las playas, estudios microbiológicos y sanitarios de la arena, entre otras investigaciones.

Los países con mayor número de publicaciones relacionadas con el tema son: Estados Unidos y Nueva Zelanda. Importante resaltar que en Latino América estas investigaciones se han desarrollado especialmente en Brasil ( 6 publicaciones), como se presenta en la Figura 3. Lo anterior permite inferir que el estudio de parásitos en playas empieza a ser un tema importante para enfrentar los problemas de salud pública, particularmente en regiones tropicales y subtropicales de países en desarrollo.

Documents by country or territory

Scopus

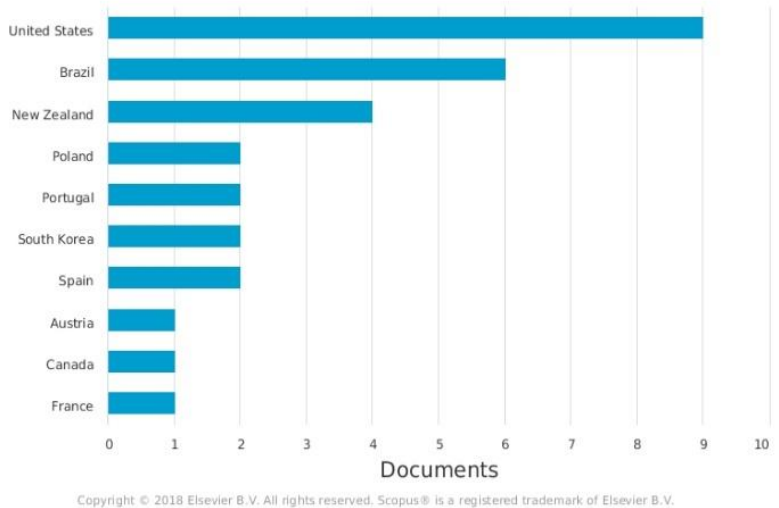

Figura 3. Países con mayor número de publicaciones relacionadas con calidad ambiental en playas. Años 2000-2018.

Fuente: Los autores a partir de www.elsevier.com. 


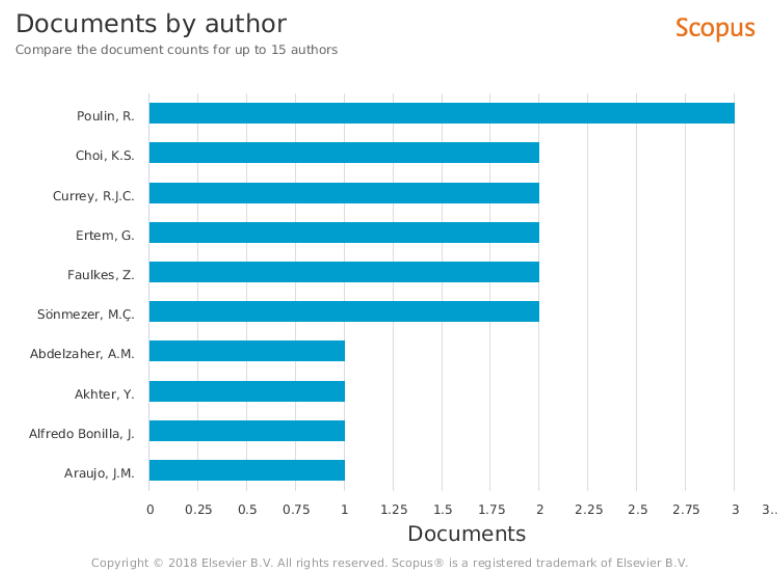

Figura 4. Autores con mayor número de publicaciones acerca de indicadores sanitarios Calidad Sanitaria ambiental de playas.

Entre los autores con mayor número de publicaciones se destacan Poulin R. con (3) tres publicaciones, Ertem G., Choi K.S, Currey R.JC., Faulkes Z., con (2) dos publicaciones y Abdelzaher Z., Araujo J.M., entre otros. con (1) una publicación (Figura 4). Lo anterior indica que hay pocas publicaciones relacionadas con el tema de parásitos en arenas como indicador de calidad ambiental de playas, lo que constituye un nicho con mucha información para realizar trabajos de investigación.

La mayoría de documentos publicados son en el 91.2\% artículos, reportados 31 artículos en una ventana de observación de 18 años, 5.9\% review y $2.9 \%$ conferencias, como se presenta en la Figura 5. Se resalta como se ha dicho en este trabajo, que es un tema de investigación cuyo interés ha estado en aumento.

Documents by type

Scopus

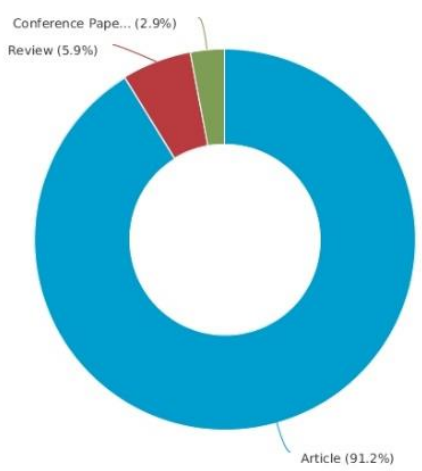

Figura 5. Tipos de publicaciones relacionadas con indicadores sanitarios en playas turísticas. Fuente: Los autores a partir de www.elsevier.com.

\section{Conclusiones}

De acuerdo a la revisión sistemática realizada, se infiere que los estudios relacionados con presencia de parásitos en arena de playas como indicadores de calidad sanitaria, han sido escasos. Los datos obtenidos de la consulta en la base de datos Scopus permiten observar que a pesar de los bajos niveles de publicaciones especializadas, es un tema de interés con tendencia promedio de 3, registrándose un máximo de cinco publicaciones en el año 2014. El país latinoamericano con mayor número de publicaciones es Brasil, con 6 documentos en una ventana de observación de 18 años.

En definitiva, la presencia de parásitos en playas se constituye en un nicho potencial para investigaciones futuras que permitan correlacionar su existencia con la calidad sanitaria de la playa e identificar el riesgo que supone para los usuarios. El campo de trabajo está en pleno crecimiento y América Latina puede jugar un papel predominante por la cantidad y calidad de sus playas.

\section{Agradecimientos}

Los autores agradecen y reconocen el trabajo de las instituciones e investigadores que forman parte del proyecto ICAPTU, especialmente a la Fundación Universitaria Tecnológico Comfenalco por ser la institución que ayudó a impulsar la investigación, además de brindar sus instalaciones y continuo acompañamiento en el proceso de la realización de este artículo. Cabe recalcar a los estudiantes que apoyan las actividades diarias de investigación en Cartagena de Indias.

\section{Literatura citada}

Acuña V. 1983. Contaminación parasitaria de los ríos callecalle, Valdivia y sus riberas. Seminario de titulación, Escuela de Tecnología Médica, Universidad Austral de Chile, Chile.

Alonso J.M., Stein M., Chamorro M.C. \& Bojanich. 2001. Contamination of soils with eggs of Toxocara in a subtropical city in Argentina. Journal of Helminthology, 75(2): $165-168$.

Ariza E., Jimenez J. \& Sardá R. 2008. Seasonal evolution of beach waste and litter during the bathing season on the Catalan coast. Waste Management, 28(12): 2604-2613. DOI: https://doi.org/10.1016/j.wasman.2007.11.012.

Barbosa M.C. \& Ferreira M. 2008. Environmental quality indicators for recreational beaches classification. Journal of Coastal Research, 246: 1439-1449. DOI: https://doi.org/10.2112/06-0901.1.

Barbosa M.C. 2006. Municipal Services on Tourist Beaches: Costs and Benefits of Solid Waste Colletion. Journal of Coastal Research, 22(5): 1070-1075.

Beltrán M., Casanova T.R. \& Velarde C. 2003. Manual de procedimientos de laboratorio para el diagnóstico de los parásitos intestinales del hombre. Serie de Normas técnicas $\mathrm{N}^{\circ}$ 37. Lima Perú. 
Benedict R. \& Neumann C. 2004. Assessing Oregon's twenty-six coastal beach areas for recreational water quality standards.Marine Pollution Bulletin, 49: 624-629.

Bordinhão P. \& Costa L.M. 2006. Areia contaminada das praias da Guanabara. Bol Comissão Meio Ambiente Assembléia Legislativa Est Rio de Janeiro.

Botero C., Pereira C. \& Manjarrez G. 2015a. Capítulo 1: Índice de Calidad Ambiental en Playas Turísticas. En: Calidad ambiental en playas turísticas aportes desde el Caribe norte colombiano. 76-88. Fundación Universitaria Jorge Tadeo Lozano, Bogota.

Botero C., Pereira C., Tosic M. \& Manjarrez G. 2015b. Design of an index for monitoring the environmental quality of tourist beaches from a holistic ap proach. Revista Ocean \& Coastal Management, 108: 65-73.

Cádiz A. 2008. Determinación de la presencia de huevos de ascárides en arenas de playas rivereñas del Río de Valdivia. Tesis M.V. Universidad Austral de Chile.

Cagilaba V. \& Rennie H.G. 2005. Literature review of beach awards and rating systems. Environmental Waikato Technical Report 2005/24. The University of Waikato, Hamilton, New Zeland.

Carrada T. 2008. Strongyloides stercoralis: Ciclo vital, cuadros clínicos, epidemiología, patología y terapéutica. Revista Latinoamericana de Patología Clínica y Medicina de Laboratorio, (55)2: 88-110.

Castillo A. \& Bermúdez M.2006. Técnicas de Diagnóstico de parásitos. Universidad de Costa Rica, Costa Rica.

Cermeño R., Arenas J., Yori J. \& Hernández I. 2008. Cryptosporidium parvum y Giardia lamblia en aguas crudas y tratadas del estado Bolívar, Venezuela. Universidad, Ciencia y Tecnología, 12(46): 39-42.

Cobas E. 2005. Parasitología veterinaria II. Universidad Nacional Agraria, Managua.

Cordero del Campiño M. \& Rojo F. 1999. Parasitología. 1 ed. McGraw-Hill Interamericana. España.

Costa M.F., Ivar Do Sul J.A., Silva-Cavalcanti J.S., Araujo M.C.B., Spengler A. \& Tpurinho P.S. 2009. On the importance of size of plastic fragments and pellets on the strandline: a snapshot of a Brazilian beach. Environmental Monitoring Assessment, 168(1-4): 1-6.

Coyne D.L., Nicol J.M. \& Claudius-Cole B. 2007. Practical plant nematology: a field and laboratory guide. SP-IPM Secretariat, International Institute of Tropical Agriculture (IITA), Cotonou, Benin.

Daprato B., Cardillo N., Kunic M., Berra Y. \& Sommerfetl I. 2011. Persistencia de la contaminación ambiental por huevos de Toxocara cati en un espacio público. Revista Española de Salud Pública, 2(35): 27-28.

Delgado Y., Enríquez D., Nuñes R. \& Pérez G. 2009. Bacterias indicadoras de contaminación fecal en aguas costeras al oeste de ciudad de La Habana, Cuba. Revista de Medio Ambiente, Turismo y Sustentabilidad, 2(2): 109-117.

Delgado Y., Miravet M.E. \& Núñez R. 2008. Indicadores microbiológicos de calidad del agua en la costa oeste de la Ciudad de La Habana. Higiene y Sanidad Ambiental, 8:387-391.

Díaz Á.M., Izquierdo C.C.S., Fernández de la Paz T. \& Izquierdo A.M. 2008. Características clínicas y epidemiológicas de las infecciones por enterococos en el niño. Rev. Cubana Pediatr., 79(1). Recuperado el 19 de enero de 2015 de: https://scielo.sld.pdf/ped/v79n1/ped08107.pdf.
Díaz C. \& Prada K. 2015. Calidad ambiental en playas turísticas aportes desde el caribe norte colombiano. Fundación Universitaria Tecnológico Comfenalco. Cartagena, Colombia.

Díaz M., Rodríguez C. \& Zhurbenko R. 2010. Aspectos fundamentales sobre el género Enterococcus como patógeno de elevada importancia en la actualidad. Revista Cubana de Higiene y Epidemiología, 48(2): 47-161.

DIGESA. 2011. Directiva sanitaria que establece el procedimiento para la evaluación de la calidad sanitaria en las playas del litoral peruano. Directiva sanitaria 1(038). Lima.

Dubinsky P., Havasiovareiterova K., Petko B., Hovorka I. \& Tomasovicova O. 1995. Role of small mammals in the epidemiology of toxocariasis. Parasitology, 110 (2): 187193.

Efstratiou M., Mavridou A. \& Richardson C. 2009. Prediction of Salmonella in seawater by total and faecal Coliforms and Enterococci. Marine Pollution Bulletin, 58(2): 201205.

Elmanama A., Ishaq M., Afifi S., Abdalallag S. \& Bahr S. 2005. Microbiological beach sand quality in Gaza Strip in comparison to seawater quality. Environmental Research, 3: $1-10$.

Elmir S., Wright M., Abdelzaher A., Solo-Gabriel H., Fleming, L., Miller G., Rybolowik M., Peter Shih M., Pillai S., Cooper J. \& Quaye E. 2007. Quantitative evaluation of bacteria released by bathers in a marine water. Water Research, 41: 3-10.

Enríquez E., Margez J.P., Alamillo M., Mejía E., Olivas J., Sosa E. \& Hernández M. 2010. Indicadores fecales y patógenos en agua descargada al río Bravo.Terra Latinoamericana, 29(4): 449-457.

EPA (U.S. Environmental Protection Agency). 1986. Ambient Water Quality Criteria for Bacteria. EPA. Washington D.C.

Espejel I., Espinoza-Tenorio A., Cervantes O., PopocaI., Mejia A. \& Delhumeau S. 2007. Proposal for an integrated risk index for the planning of recreational beaches: use at seven Mexican arid sites. Journal of coastal research, 50 (51): 47-51.

Espindula J. 2004. Caracterização Bacteriológica E Fisicoquímica Das Águas Do Aquiífero Freático Do Cemitério Da Varzea-Recife. Dissertação de Mestrado. Universida de Federal de Pernambuco. Centro de tecnología e Geociências. Pós-GraduaçaoemGeociencias, Brasil.

Fisher M. 2003. Toxocaracati: an under estimated zoonotic agent. Trends in Parasitolgy, (19)4: 167-170.

Fredes G. 2015. Detección y caracterización de Cryptosporidium spp. mediante métodos tradicionales y PCR en diferentes matrices (heces y aguas). Programa de Doctorado en Biociencias y Ciencias Agroalimentarias, Córdoba, España.

Gavio B., Palmer-Cantillo S. \& Mancera J.E. 2010.Historical analysis (2000-2005) of the coastal water quality in San Andrés Island, SeaFlower Biosphere Reserve, Caribbean Colombia. Marine Pollution Bulletin, 60(7): 1018-1030.

Girard de Kaminskyrina. 2003. Manual de parasitología. Métodos para Laboratorios de Atención Primaria de Salud. 2da. Organización Panamericana de la Salud (OPS), Organización Mundial de la Salud (OMS) y la Universidad Nacional Autónoma de Honduras (UNAH). 
Glickman L. 1993. The epidemiology of human toxocariasis.Toxocara and Toxocariasis, Clinical, Epidemiological and Molecular Perspectives. Institute of Biology and the British Society for Parasitology, 23(31): 3-10.

González A.M., Paranhos R. \& Lutterbach M.S. 2010. Relationships between fecal indicators and pathogenic microorganisms in a tropical lagoon in Rio de Janeiro, Brazil. Environmental Monitoring and Assessment, 164B(1): 207-219.

González y Cáceres A.P., Gonçalves F, Cazoria I. \& Carvalho S. 2005. Contaminação do solo porhelmintos de importância médica na praia do sul (Milionários), Ilhéus - BA / Contamination of soil by helmints of medical importance in the south beach (Milionários) Ilhéus - BA. Revista brasileira de análisis clínicas. Recuperado de: http://bases.bireme.br/cgi-

bin/wxislind.exe/iah/online/?IsisScript=iah/iah.xis\&src= google\&base=LILACS\&lang=p\&nextAction=lnk\&expr Search=411937\&indexSearch=ID.

Guerrero A., García M. \& Ramon C.J. 2013. Enteroparasitos en arena de playa como indicadores de contaminación fecal y su relación con condiciones ambientales en playas Quizandal, Puerto Cabello. Academia Biomédica Digital. Venezuela.

Guerrero A., Quiñones M.V., Sequera E.J. \& Marín J.L. 2014. Parásitos patógenos en arena de playa y su relación con condiciones ambientales, en un balneario de Puerto Cabello, Venezuela, 2012-2013. Boletín de malariología y salud ambiental. Agosto-Diciembre. LIV, (2): 150-158.

Herrera A. \& Suárez P. 2005. Indicadores bacterianos como herramientas para medir la calidad ambiental del agua costera. INCI. 30(3): 171-176. Recuperado de: http://www.who.int/es/.

Hurtado Y., Botero C., \& Herrera E. 2009. Selección y propuesta de parámetros para la determinación de la calidad ambiental en playas turísticas del Caribe Colombiano. Revista Ciencia en su PC, 4: 42-53.

Kitchenham. 2004. Procedures for Performing Systematic Reviews. Keele University Technical Report TR/SE0401. Keele University, Newcastle, Reino Unido.

Laggagio V.R.A., Jorge L.L., Oliveira V., Flores M.L. \& Silva J.H. 2001. Presença de endoparasitas em três praias do Município de Guaíba-RS/Brasil. Instituto Brazilero de recursos avanzados. Recuperado el 15 de julio de 2005 de: http://www.redevet.com.br/artigos/praias.html.

Larrea J., Rojas M., Heydrich M., Romeu B., Rojas N. \& Lugo D. 2009. Evaluación de la calidad microbiológica de las aguas del Complejo Turístico Las Terrazas, Pinar del Río (Cuba). Higiene y Sanidad Ambiental, 9: 492504.

Lescano S.A., Chieffi P.P., Peres B.A., De Mello E.O., Velarde C. N., Salinas A. A. \& Rojas C. E. 1998. Soil contamination and human infection by Toxocara $\mathrm{sp}$. in the urban area of Lima, Peru. Memórias do Instituto Oswaldo Cruz, (93)6: 733-734.

Ludlam K.E. \& Platt T.R. 1989. The relationship of park maintenance and accesibility to dogs to the presence of Toxocara spp. ova in the soil. American Journal of Public Health, (79)5: 633-634.
Madrid V., Sardella N.H., Denegri G. \& Hollmann P. 2005. Contaminación de playas de la ciudad de Mar del Plata con parásitos de importancia sanitaria. Laboratorio de Zoonosis Parasitarias. Facultad de Ciencias Exactas y Naturales. Universidad Nacional del Mar de Plata. Argentina.

Magnaval J.F., Glickman L.T., Dorchies P. \& Morassin B. 2001. Highlights of human toxocariasis. The Korean Journal of Parasitology, 39(1): 1-11.

Mansilha C.R., Coelho C.A., Heitor A. M., Amado J., Martins J.P. \& Gameiro P. 2009. Bathing waters: New directive, new standards, new quality approach. Marine Pollution Bulletin, 58: 1562-1565.

Marchand E.O. 2002. Microorganismos indicadores de la calidad del agua de consumo humano en Lima Metropolitana. Tesis para optar al título profesional de Biólogo con mención en Microbiología y Parasitología. Universidad Nacional Mayor de San Marcos. Lima, Perú. Mariano J.M., Sánchez G., Martínez B., Spinola E. \& Redondo R. 2012. Comparación de dos nuevas técnicas de sedimentación y métodos convencionales para la recuperación de parásitos intestinales. Laboratorio de Parasitología, Laboratorio Clínico, Hospital Infantil de México. Revista Latinoamericana de Patología Clínica y Medicina de Laboratorio, 59(4): 233-242. Recuperado de: http://www.medigraphic.com/ patologiaclinica.

Martínez F.A. 1979.Determinación de larvas de Ancylostoma sp. en las playas de la ciudad de Corrientes. Revista Veterinaria, 2(2): 39-47.

Mejía E. 2010. Determinación del Dipylidium caninum a través del método de sedimentación en caninos de 1 mes a un año de edad, en la parroquia la Magdalena del distrito metropolitano de Quito. Universidad Técnica de Cotopaxi.Latacunga, Ecuador.

Méndez R.I., San Pedro L., Castillo E.R. \& Vázquez E.B. 2010. Modelación del tiempo de conservación de muestras biológicas de agua. Revista Internacional de Contaminación Ambiental, 26(4): 327-335.

Milano A.M.F. \& Oscherov E.B. 2002. Contaminación de playas de la ciudad de Corrientes con parásitos caninos capaces de infectar al hombre. Parasitología Latinoamericana, 57(3-4): 119-123.

Ministerio de Vivienda, Ciudad y Territorio. 2012. Reglamento Técnico del Sector de Agua Potable y Saneamiento Básico: TíTUlO F. Sistemas de Aseo Urbano. Viceministerio de Agua y Saneamiento Básico. Bogotá, D.C. Colombia.

Morales M. \& Esquivia V. 2014. Contaminación de playas turísticas de la ciudad de Cartagena de Indias con parásitos de importancia sanitaria 2012-2014. Tesis de Maestría. Universidad San Buenaventura. Cartagena.

Morgan R. 1999. A novel, user-based rating system for tourist beaches. Tourism Management, 20(4): 393-410.

Nelson K. 2003. Concentrations and inactivation of Ascaris eggs and pathogen indicator organisms in wastewater stabilization pond sludge. Water science and technology, 48: 89-95.

Oigman-Pszczol S.S. \& Creed J.C. 2007.Quantification and classification of marine litter on beaches along Armacao dos Buzios, Rio de Janeiro, Brazil. Journal of Coastal Research, 23(2): 421-42.

Orellana J. 2005. Ingeniería Sanitaria- UTN - FRRO. Unidad Temática $\mathrm{N}^{\circ} 3$. Características del agua potable. UTN. Argentina. 
Ortigoza S. \& Cruz M. 2012. Manual de procedimientos para el laboratorio de la E. E. Universidad veracruzana. Facultad de bioanalisis región veracruz. Venezuela.

Overgaauw P.A. 1997. Aspects of Toxocara epidemiology: Toxocariasis in dogs and cats. Clinical microbiology reviews, 23(3): 233-251.

Passucci J.A. \& West M. 1996. Parasitosis interna en un albergue de perros en la ciudad de Tandil 1995. Pet's, 12(67): 473-475.

Phillips M., Solo/Gabriele H., Piggot A., Klaus J. \& Zhang, Z. 2011. Relationships between sand and water quality at recreational beaches. Water Research, 45(20): 67636769.

Pichard D.C., Hensley J.R., Williams E., Apolo A.B., Klion A.D. \& DiGiovanna J.J. 2014. Rapid development of migratory, linear, and serpiginous lesions in association with immunosuppression. Journal of the American Academy of Dermatology, 70(6): 1130-1134.

Polo L.J. 2006. Determinación de la contaminación de los suelos de los parques públicos de la localidad de suba, Bogotá D.C con nemátodos gastrointestinales de importancia zoonótica. Tesis de Maestría en Salud Pública. Universidad Nacional de Colombia, Bogotá.

Pulido M., De Navia L., Torres M. \& Prieto A. 2005. Indicadores microbiológicos de contaminación de las fuentes de agua. NOVA Publicación en Ciencias Biomédicas, 3(4): 69-79.

Pullés M. 2014. Microorganismos indicadores de la calidad del agua potable en Cuba. Revista CENIC Ciencias Biológicas, 45(1): 25-36.

Rocca E., Riera C., Míriam V., Fragell R. \& Junyent R. 2008. A combined assessment of beach occupancy and public perceptions of beach quality: A case study in the Costa Brava, Spain. Ocean \& Coastal Management, 51: 839846.

Rodríguez R. \& Cob L. 2005. Técnicas Diagnósticas en Parasitología Veterinaria. Segunda edición. Universidad Técnica de Yucatán, México.

Roldán G. 2003. Bioindicación de la calidad del agua en Colombia: uso del método BMWP/Col. Universidad de Antioquía, Medellín, Colombia.

Sabino R., Rodrigues R., Costa I., Carneiro C., Cunha M., Duarte A., Faria N., Ferreira F.C., Gargaté M.J., Júlio C., Martins M.L., Neversi M.B., Oleastro M., Solo-Gabrielej H., Veríssimo C., Viegas C., Whitman R.L. \& Brandão J. 2013. Routine screening of harmful microorganisms in beach sands: Implications to public health. Science of the Total Environment, 472: 1603-1609.

Salcedo H.G.D. 2013. Calidad del agua de la Bahía de Cartagena en relación con la distribución espacial de Coliformes totales, Escherichia coli y Enterococcus sp. durante la temporada seca del 2013. Universidad de San Buenaventura. Cartagena de Indias.

Santiago-Rodriguez T.M., Tremblay R.L., ToledoHernandez C., Gonzalez-Nieves J.E., Ryu H., Santo Domingo J.W. \& Toranzos G.A. 2012. Microbial quality of tropical inland waters and effects of rainfall events. Environmental Microbiology, 78(15): 5160-5169.
Schapiro J., Eddi C., Caracostantogolo J., Peña M., Cutelle C. \& Castañor. 2001. Presencia de huevos de enteroparásitos zoonóticos en espacios públicos de la ciudad de Pilar. III Congreso Argentino y II Congreso Latinoamericano de zoonosis. Ciudad Autónoma de Buenos Aires.

Shimizu T. 1993. Prevalence of Toxocara eggs in sandpits in Tokushima city and its outskirts. Journal of Veterinary Medical Science, 55(5): 807-811.

Silva J.B. 2008. Flag Items as a Tool for Monitoring Solid Waste from Users on Urban Beaches. Journal of Coastal Research, 24(4): 890-898.

Steiner T., Thielman N. \& Guerrant R. 1997. Protozoal agents. What are the dangers for the public water-supply? Annual review of medicine, 48: 329-340.

Tudor D. \& Williams A. 2008. Important Aspects of Beach Pollution to Managers: Wales and the Bristol Channel, UK. Journal of Coastal Research, 24(3): 735-745.

Van den Berghe E., De Winter T. \& De Vuyst L. 2006. Enterocin A production by Enterococcus faecium FAIRE 406 is characterised by a temperature- and $\mathrm{pH}-$ dependent switch-off mechanism when growth is limited due to nutrient depletion. International journal of food microbiology, 107(2): 159-170.

Vandermeulen H. \& Cobb D. 2004. Marine environmental quality: a Canadian history and opinions for the future. Ocean \& Coastal Management, 47: 243-256.

Vergaray G., Méndez C.R., Morante H.Y., Heredia V.I. \& Béjar V.R. 2007. Enterococcus y Escherichia coli como indicadores de contaminación fecal en playas costeras de Lima. Revista del Instituto de Investigación de la Facultad de Ingeniería Geológica, Minera, Metalúrgica y Geográfica, 10(20): 6-82.

Vogel C., Rogerson A., Schatz S., Laubach H., Tallman A. \& Fell J. 2007. Prevalence of yeasts in beach sand at three bathing beaches in South Florida. Water Research, 41(9): 1915-1920.

WHO \& UNICEF (World Health Organization \& UNICEF). 2005. Water for life. Making it happen. WHO, Washington, USA.

WHO. 2012. International travel and health. World Health Organisation. Ginebra. Recuperado 24 de febrero de 2016 de: http://www.who.int/ith/ITH_EN_2012_WEB_1.2.pdf.

WHO. 2014. Enfermedades transmitidas por vectores. Nota descriptiva $\mathrm{N}^{\circ} 387$, World Health Organisation. Ginebra. Recuperado 24 febrero de 2016 de: http://www.who.int/mediacentre/factsheets.

Yamahara Ç. K.M., Walters S.P. \& Boehm A.B. 2009. Growth of Enterococci in unaltered, unseeded beach sands subjected to tidal wetting. Microbial Ecology, 75(6): 1517-1524.

Zhang W., Wang J., Fan J., Gao D. \& Ju H. 2013. Effects of rainfall on microbial water quality on Qingdao No. 1 Bathing Beach, China.Marine Pollution Bulletin, 66(1-2): $185-190$. 
Tabla 2. Estudios relevantes sobre parásitos en playas en diferentes países de Latinoamérica.

\begin{tabular}{|c|c|c|c|}
\hline AÑO & PAÍS & PLAYAS & DETALLES \\
\hline $1979-2002$ & Argentina & 4 Playas de Corrientes & $\begin{array}{l}\text { Reportaron la presencia de Ancylostoma sp. en } 1979 \text {, con } \\
\text { prevalencias entre } 11.1 \% \text { y } 38 \% \text {. (Martínez, 1979). En el 2002, } \\
\text { Milano \& Oscherov realizaron un estudio en estas mismas } \\
\text { playas registrando prevalencia parasitarias de } 37.5 \% \text {. }\end{array}$ \\
\hline $1983-2008$ & Chile & $\begin{array}{l}4 \text { playas Collico, Las } \\
\text { Animas, Las Mulatas } \\
\text { e Isla Teja }\end{array}$ & $\begin{array}{l}\text { Se encontraron en } 1983 \text { huevos Ascaris sp. y T. canis (Acuña, } \\
\text { 1983). En el 2008, en estas mismas playa se recolectaron } 36 \\
\text { muestras de arena, de las cuales una sola se encontró positiva, } \\
\text { lo que difiere de los resultados obtenidos por Milano \& } \\
\text { Oscherov (2002) (Cádiz, 2008). }\end{array}$ \\
\hline \multirow[t]{2}{*}{$2001-2004$} & Brasil & $\begin{array}{l}\text { Playas de Guaiba (1) y } \\
\text { Guanabara (2) }\end{array}$ & $\begin{array}{l}\text { a) Se demostraron niveles elevados de indicadores fecales y } \\
\text { parásitos en la arena de playas de Guaíba, Río Grande del Sur, } \\
\text { con la presencia de huevos de Taeniasp. ( } 3.48 \% \text { ), quistes de } \\
\text { Giardia sp. }(9.0 \% \text { ) y una baja prevalencia de Ancylostoma } \\
\text { (Laggagio et al., 2001). }\end{array}$ \\
\hline & & & $\begin{array}{l}\text { b) Presentaron Ascaris lumbricoides, Enterobius vermicularis } \\
(30 \%),(20 \%) \text {, quistes de Giardia lamblia (16\%), quistes } \\
\text { Entamoeba histolytica (16\%), Strongyloides stercoralis (10\%), } \\
\text { Ancylostomids (5\%), Trichuris trichiura(5\%) (Bordinhão \& } \\
\text { Costa, 2006). }\end{array}$ \\
\hline $2013-2014$ & Venezuela & $\begin{array}{l}\text { Puerto } \\
\text { Quizanda }\end{array}$ & $\begin{array}{l}\text { Mostraron que el } 25 \% \text { de las muestras presentaban } \\
\text { contaminación por parásitos patógenos, corroborando que la } \\
\text { playa estudiada no cuenta con condiciones propicias de } \\
\text { saneamiento ambiental para visitas turísticas(Guerrero et al., } \\
2013 \text { ). El } 2014 \text { se realizó una investigación descriptiva desde } \\
\text { marzo de } 2013 \text { a enero de } 2014 \text {, encontrándose parásito de } \\
\text { interés clínico-epidemiológico, dando como resultado que el } \\
10.4 \% \text { de las muestras fueran positivas para patógenos que } \\
\text { corresponden a un 50\% de larvas de Strongyloides stercoralis, } \\
\text { seguidos con } 37.5 \% \text { de larvas de Anquilostomideos y un } 12.5 \% \\
\text { de huevos de este mismo grupo de parásitos; además, no se } \\
\text { encontró ninguna relación estadística entre las estaciones } \\
\text { lluvia/sequías (Guerrero et al., 2014). }\end{array}$ \\
\hline 2014 & Cartagena & $\begin{array}{l}\text { Boquilla, Bocagrande } \\
\text { y Punta Arena }\end{array}$ & $\begin{array}{l}\text { En orden de frecuencia, el } 50.21 \% \text { de los parásitos encontrados } \\
\text { correspondió a Toxocara sp., seguido por el } 25.51 \% \text { de } \\
\text { Ancylostoma sp. El sector de playa más contaminado fue } \\
\text { Boquilla } 1 \text {, seguido de Boquilla2, siendo los parásitos más } \\
\text { frecuente el Toxocara sp. y Strongyloides sp., respectivamente; } \\
\text { así mismo, en la playa de Punta Arena se encontró de manera } \\
\text { significativa más Strongyloides sp. que en Bocagrande y la } \\
\text { Boquilla. Las condiciones climáticas tienen influencia sobre el } \\
\text { crecimiento delos parásitos. Las altas temperaturas de la época } \\
\text { de sequía favorecen el crecimiento de Strongyloides sp. y } \\
\text { Toxocara sp., y disminuyen el de Ancylostomasp. (Morales \& } \\
\text { Esquivia, 2014). }\end{array}$ \\
\hline
\end{tabular}

\footnotetext{
${ }^{1}$ Grupo de Investigación Ambiental GIA, Fundacion Universitaria Tecnologico Comfenalco - Cartagena, ID: 60110627.gmanjarrez@tecnologicocomfenalco.edu.co.

${ }^{2}$ Grupo de Investigación GIA, Fundación Universitaria Tecnológico Comfenalco, Cartagena de Indias, Colombia. Jorgeivan80@ hotmail.com.

${ }^{3}$ Grupo de Investigación GIA, Fundación Universitaria Tecnológico Comfenalco, Cartagena de Indias, Colombia. gonzalezpg30@gmail.com.

${ }^{4}$ Grupo de Investigación en Sistemas Costeros, Colombia. playascol@gmail.com.

${ }^{5}$ Grupo de Investigación GIA, Fundacion Universitaria Tecnologico Comfenalco - Cartagena. ID: 60110627. cdiaz@tecnologicocomfenalco.edu.co.
} 